\title{
Effect of Sensing Film Thickness on Sensing Characteristics of Dual-Gate Poly-Si Ion-Sensitive Field-Effect-Transistors
}

\author{
Li-Chen Yen, Ming-Tsyr Tang, Chia-Ying Tan, Tung-Ming Pan, and Tien-Sheng Chao
}

\begin{abstract}
We investigate the effect of sensing film thickness on the sensing characteristics of dual-gate (DG) poly-Si ion-sensitive field-effect transistors (ISFETs). The pH sensitivity (from 37.57 to $9.32 \mathrm{mV} / \mathrm{pH}$ ) of the DG poly-Si ISFET device degrades with the increase in the sensing film thickness (from 20 to $120 \mathrm{~nm}$ ), whereas hysteresis voltage (from 6.7 to $1.12 \mathrm{mV}$ for a neutral to acid to alkaline to neural loop) and drift rate (from 13.47 to $<3 \mathrm{mV} / \mathrm{h}$ at $\mathrm{pH}$ 7) improve accordingly. An improved hysteresis and drift phenomena are attributed to the reduction in top-gate capacitance of the sensing membrane, causing a smaller capacitive-coupling ratio (top-gate capacitance of sensing membrane to bottom-gate capacitance of tetraethylorthosilicate oxide).
\end{abstract}

Index Terms-Drift, dual-gate (DG), hysteresis, ion-sensitive field-effect transistor (ISFET), poly-Si, sensitivity.

\section{INTRODUCTION}

$\mathbf{T}$ HE ion-sensitive field-effect transistor (ISFET) was first introduced by P. Bergveld in 1970 to replace the fragile glass electrode for the measurement of the $\mathrm{pH}$ and concentration of ions [1]. According to the operation principle of ISFET, different $\mathrm{pH}$ concentrations will induce various surface potentials at the sensing membrane, leading to the variation of threshold voltage $\left(\mathrm{V}_{\mathrm{TH}}\right)$. The development of high-dielectric constant (high- $\kappa$ ) gate insulator materials has recently become a topic of considerable interest in ISFETs because of their high sensitivity [2]-[4]. However, most of these insulator materials exhibit less than the Nernstian $\mathrm{pH}$ sensitivity of $59.5 \mathrm{mV} / \mathrm{pH}$ [2], [3], which is also the maximum achievable sensitivity. In contrast, the dual-gate (DG) ISFET could exceed the Nernstian $\mathrm{pH}$ sensitivity due to the asymmetric gate oxide thicknesses (top-gate and bottom-gate oxide thicknesses) [5]. The $\mathrm{pH}$ response of the DG ISFET was determined as a function of the capacitive coupling. The sensitivity could be enhanced by one order of magnitude [5], [6].

Manuscript received August 14, 2014; revised September 8, 2014, September 29, 2014, and October 19, 2014; accepted October 20, 2014. Date of current version November 20, 2014. This work was supported by the National Science Council of Taiwan under Grant NSC100-2221-E-009012-MY3. The review of this letter was arranged by Editor A. Flewitt.

L.-C. Yen, M.-T. Tang, and T.-S. Chao are with the Department of Electrophysics, National Chiao Tung University, Hsinchu 30010, Taiwan.

C.-Y. Tan and T.-M. Pan are with the Department of Electronics Engineering, Chang Gung University, Taoyuan 33302, Taiwan (e-mail: tmpan@mail.cgu.edu.tw).

Color versions of one or more of the figures in this letter are available online at http://ieeexplore.ieee.org.

Digital Object Identifier 10.1109/LED.2014.2365478
Nevertheless, very few studies have addressed the non-ideal electrical characteristics of the DG ISFET, such as hysteresis and drift phenomena. In this letter, we explore the effect of sensing film thickness on the sensing characteristics ( $\mathrm{pH}$ sensitivity, hysteresis width and drift rate) of DG poly-Si ISFET devices. We find that the $\mathrm{pH}$ sensitivity of the DG polySi ISFET decreases as the sensing film thickness increases. Conversely, the hysteresis voltage and drift rate improve accordingly.

\section{EXPERIMENTAL}

The fabrication of an ISFET device was started by a $500 \mathrm{~nm}$ thick thermal oxide layer grown on a Si wafer at $980{ }^{\circ} \mathrm{C}$. The phosphorus-in-situ doped polycrystalline-Si (poly-Si) layer (150 nm thick) was deposited using a low-pressure chemical vapor deposition (LPCVD) system at $550{ }^{\circ} \mathrm{C}$. Next, a reactive ion etching process was used to define the bottomgate structure of the device. The deposition of the $25 \mathrm{~nm}$ thick tetraethylorthosilicate (TEOS) oxide as a gate dielectric was performed using the LPCVD system. Then, a $50 \mathrm{~nm}$ thick amorphous silicon $(\alpha-\mathrm{Si})$ layer was deposited by LPCVD at $550{ }^{\circ} \mathrm{C}$ and subsequently converted into a poly-Si film as a channel layer using a solid-phase crystallization process at $600{ }^{\circ} \mathrm{C}$ for $24 \mathrm{~h}$ in a $\mathrm{N}_{2}$ ambient. Arsenic ions were implanted into the source/drain (S/D) region using a $36 \mathrm{keV}$ ion energy at a dose of $5 \times 10^{15} \mathrm{~cm}^{-2}$. After the lithography process, the implanted dopants were activated. Afterwards, $600 \mathrm{~nm}$ thick Al pads were grown using a physical vapor deposition system followed by wet etching of the Al film. Due to the appearance of a large source-drain leakage current when used in various $\mathrm{pH}$ solutions [7], a $200 \mathrm{~nm}$ thick oxynitride passivation layer was deposited by PECVD. The sensing area was opened subsequently with the lithography process and followed by wet etching of the oxide film. 20, 70 and $120 \mathrm{~nm}$ thick TEOS oxide films as sensing membranes were deposited by PECVD. A $\mathrm{H}_{2}$ sinter process at $300{ }^{\circ} \mathrm{C}$ for $30 \mathrm{~min}$ was adopted for the passivation of the defect and dangling bonds between the oxide and poly-Si channel interface. Finally, the microfluidic channel was set up and the $\mathrm{pH}$ sensing measurement can be implemented. The cross-sectional view of the DG poly-Si ISFET device is shown in Fig. 1(a).

For the steady $\mathrm{pH}$ responses, all DG-ISFET devices were immersed in deionized water for $12 \mathrm{~h}$ [8]. The drain current versus gate voltage $(\mathrm{I}-\mathrm{V})$ curves for various $\mathrm{pH}$ buffer solutions were measured using an Agilent B1500A semiconductor 


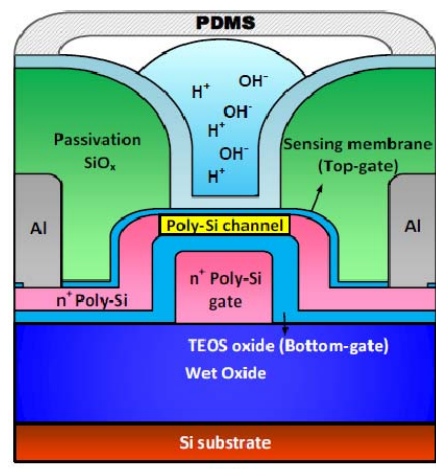

(a)

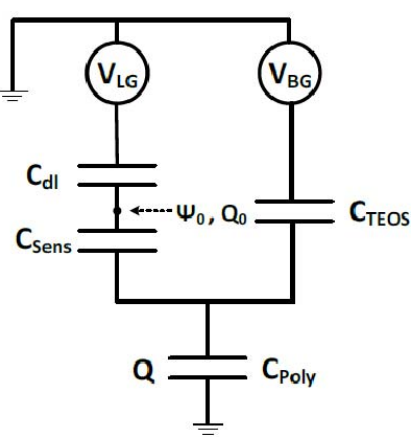

(b)
Fig. 1. (a) The cross-sectional view of the double-gated poly-Si ISFET device. (b) In equilibrium when no source-drain voltage is applied, the charge states are defined by the capacitors shown in this equivalent circuit. $C_{d l}$ denotes the electrolyte double-layer capacitance, $C_{S e n s}$ is the top-gate capacitance of sensing membrane, $C_{P o l y}$ denotes the capacitance of the inversion layer in poly-Si channel, $C_{T E O S}$ is the bottom-gate capacitance of TEOS oxide, $Q$ stands for the induced charge in the poly-Si channel, and $Q_{0}$ and $\psi_{0}$ represent the charge and potential at the oxide-electrolyte interface, respectively.
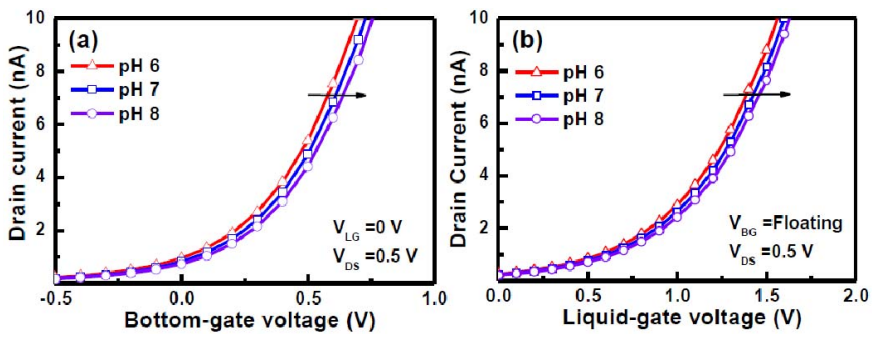

Fig. 2. The transfer characteristics of the DG poly-Si ISFETs with $20 \mathrm{~nm}$ sensing membrane measured various $\mathrm{pH}$ solutions from $\mathrm{pH} 6$ to $\mathrm{pH} 8$ and operated under (a) bottom-gate and (b) liquid-gate modes.

parameter analyzer and a commercial $\mathrm{Ag} / \mathrm{AgCl}$ reference electrode. In order to avoid the light and noise interference, electrical measurement was performed in a dark box. The effective capacitance network is shown in Fig. 1(b). The various capacitances are the double-layer capacitance $C_{d l}$ at the electrolyte-oxide interface, the top-gate capacitance of the sensing membrane $C_{S e n s}$ on top of the poly-Si channel, the bottom-gate capacitance of TEOS oxide $C_{T E O S}$ at bottom of the poly-Si channel, and the inversion layer capacitance $C_{P o l y}$.

\section{RESUlTS AND DISCUSSION}

Figs. 2(a) and (b) show typical I-V characteristics of the DG ISFETs operated at liquid-gate mode (bottom-gate voltage floating, drain voltage of $0.5 \mathrm{~V}$ and liquid-gate sweep) and bottom-gate mode (bottom-gate sweep, drain voltage of $0.5 \mathrm{~V}$ and liquid-gate grounded) for different $\mathrm{pH}$ solutions (pH 6, 7, 8), respectively. The I-V curve shifts to a more positive threshold voltage value with increasing the $\mathrm{pH}$ value due to the sensing membrane being exposed to negatively charged hydroxyl $(-\mathrm{OH})$ groups. Hence, the change in the surface charge density caused by the variation of $\mathrm{pH}$ value can be described by the site-binding model [9], considering the protonation and de-protonation process of $\mathrm{OH}$ groups.

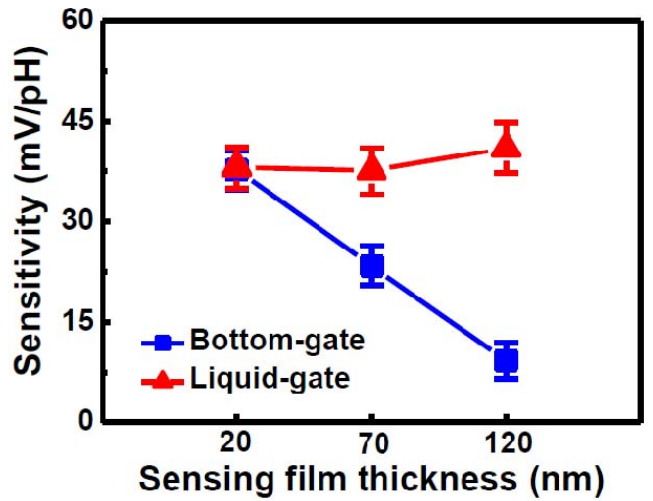

Fig. 3. The sensitivities of the DG poly-Si ISFETs with 20, 70 and $120 \mathrm{~nm}$ sensing membranes operated under liquid-gate and bottom-gate modes.
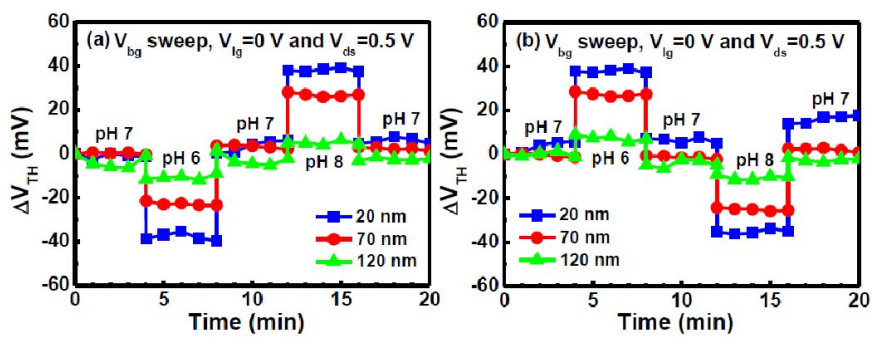

Fig. 4. The hysteresis curves of the poly-Si ISFETs using 20, 70 and $120 \mathrm{~nm}$ sensing membranes operated under bottom-gate mode and measured in $\mathrm{pH}$ loops of (a) $\mathrm{pH} 7 \rightarrow 6 \rightarrow 7 \rightarrow 8 \rightarrow 7$ and (b) $\mathrm{pH} 7 \rightarrow 8 \rightarrow 7 \rightarrow 6 \rightarrow 7$.

Fig. 3 presents the pH sensitivity of the DG ISFETs with different sensing membranes operated under liquid-gate and bottom-gate modes. The measured sensitivities of DG ISFETs under liquid-gate mode were $37.68,38.04$ and $41 \mathrm{mV} / \mathrm{pH}$ for 20,70 and $120 \mathrm{~nm}$ sensing membranes, respectively. The $\mathrm{pH}$ sensitivity of the liquid-gate mode is independent of the sensing membrane thickness. According to the site-binding model, the threshold voltage shift of ISFET is primarily attributed to an interfacial potential at the insulator-electrolyte interface [10], indicating that the sensitivity of the liquid-gate mode mainly depends on the material of the sensing membrane. On the other hand, the $\mathrm{pH}$ sensitivities of the DG ISFETs with 20,70 and $120 \mathrm{~nm}$ sensing membranes operated under bottom-gate mode are 37.57, 23.4 and $9.32 \mathrm{mV} / \mathrm{pH}$, respectively. The $\mathrm{pH}$ sensitivity operated under bottom-gate mode decreases with increasing the sensing membrane thickness. The decreased sensitivity could be explained by the classical coupling relation [6]:

$$
\Delta V_{T H, B G}=\frac{C_{\text {Sens }}}{C_{T E O S}} \Delta V_{T H, L G}=-\frac{C_{\text {Sens }}}{C_{T E O S}} \Delta \psi_{0}
$$

where $\psi_{0}$ is the surface potential. In comparison with a regular ISFET, the change in threshold voltage is modified by the capacitive coupling $C_{\text {Sens }} / C_{T E O S}$. The sensitivity degradation of ISFET under bottom-gate mode may be attributed to the decrease in the $C_{\text {Sens }}$ (or increase in the sensing membrane thickness).

Figs. 4(a) and (b) show the hysteresis effect of the DG ISFETs with different sensing membranes measured for the 


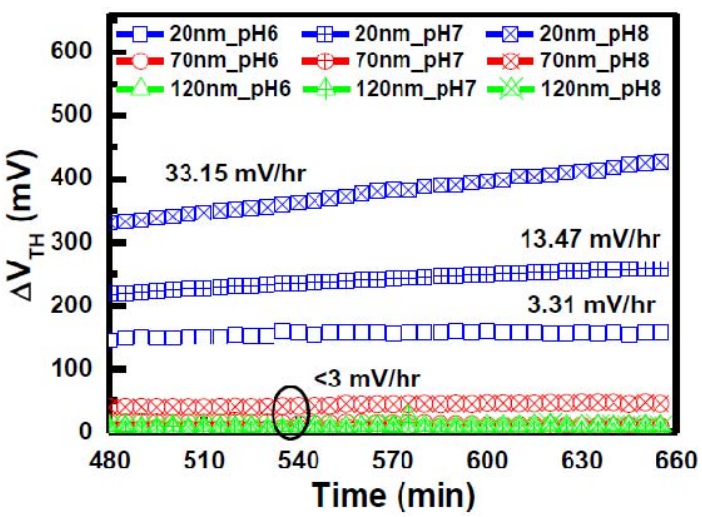

Fig. 5. Drift rates of the DG ISFETs using 20, 70 and $120 \mathrm{~nm}$ sensing membranes operated under bottom-gate mode and measured in solutions at pH 6, 7 and 8 .

following pH loops: a $7 \rightarrow 6 \rightarrow 7 \rightarrow 8 \rightarrow 7$ cycle (acid-first cycle) and a $7 \rightarrow 8 \rightarrow 7 \rightarrow 6 \rightarrow 7$ cycle (base-first cycle), respectively. It is found that the hysteresis widths of 20,70 and $120 \mathrm{~nm}$ sensing membranes measured in the acid-first are 6.7, 2.01 and $1.12 \mathrm{mV}$, while the base-first cycle are 12.73, 2.39 and $2.44 \mathrm{mV}$. The results show the asymmetric hysteresis between the acid-first and base-first cycles. A hydrogen ion $\left(\mathrm{H}^{+}\right)$is attracted to the negative end of a polar water molecule and bonds with it to form a hydronium ion $\left(\mathrm{H}_{3} \mathrm{O}^{+}\right)$, which is quite stable. The $\mathrm{H}_{3} \mathrm{O}^{+}$represents a $\mathrm{H}^{+}$in aqueous solution. The $\mathrm{OH}^{-}$ions are smaller in size than the $\mathrm{H}_{3} \mathrm{O}^{+}$ions, hence, the diffusion rates of the $\mathrm{OH}^{-}$ions into the buried sites of the sensing membrane are faster than the $\mathrm{H}_{3} \mathrm{O}^{+}$groups, leading to an asymmetric hysteresis phenomenon [11]. In addition, the hysteresis width decreases as the sensing membrane thickness increases. The results indicate that the increase in sensing membrane thickness causes a smaller capacitive-coupling ratio $\left(C_{S e n S} / C_{T E O S}\right)$. As a consequence, the hysteresis phenomenon of an ISFET device using a sensing membrane thickness over $70 \mathrm{~nm}$ performed under the bottom-gate mode becomes insignificant.

Fig. 5 shows the long-term response of the DG ISFET devices fabricated at different sensing membrane thicknesses and measured in different $\mathrm{pH}$ values under bottom-gate mode. The formation of $\mathrm{Si}-\mathrm{OH}$ buried sites occurred at the oxide surface because of the penetration of ions into the oxide film. Therefore, the change in the oxide charge leads to a decrease in the insulator capacitor with time, which could cause a temporal increase in the threshold voltage [12], [13]. The drift rate increases with increasing the $\mathrm{pH}$ value, which is consistent with previous reports [14]-[16]. Furthermore, the drift behavior is also reduced when the sensing membrane becomes thick, suggesting a decrease in the $C_{\text {Sens }} / C_{T E O S}$ ratio. Consequently, the drift behavior under the bottom-gate mode becomes insignificant when the sensing membrane thickness is more than $70 \mathrm{~nm}(<3 \mathrm{mV} / \mathrm{hr})$.
In practical applications of a DG poly-Si ISFET, a trade-off between sensitivity and non-ideal effect should be considered.

\section{Conclusion}

We investigated the $\mathrm{pH}$ sensitivity of a DG poly-Si ISFET using various sensing film thicknesses performed under liquid-gate and bottom-gate modes. The non-ideal phenomena of hysteresis and drift were also evaluated. It is found that the $\mathrm{pH}$ sensitivity of the DG poly-Si ISFET increases as the sensing film thickness decreases, while hysteresis voltage and drift rate increase accordingly. This behavior is attributed to the increase in the $C_{S e n s} / C_{T E O S}$ ratio.

\section{REFERENCES}

[1] P. Bergveld, "Development of an ion-sensitive solid-state device for neurophysiological measurements," IEEE Trans. Biomed. Eng., vol. BME-17, no. 1, pp. 70-71, Jan. 1970.

[2] C. N. Tsai et al., "Study on the sensing characteristics and hysteresis effect of the tin oxide pH electrode," Sens. Actuators B, Chem., vol. 108, nos. 1-2, pp. 877-882, Jul. 2005.

[3] S. Yoshida, N. Hara, and K. J. Sugimoto, "Development of a wide range $\mathrm{pH}$ sensor based on electrolyte-insulator-semiconductor structure with corrosion-resistant $\mathrm{Al}_{2} \mathrm{O}_{3} \quad \mathrm{Ta}_{2} \mathrm{O}_{5}$ and $\mathrm{Al}_{2} \mathrm{O}_{3} \quad \mathrm{ZrO}_{2}$ double-oxide thin films," J. Electrochem. Soc., vol. 151, no. 3, pp. H53-H58, Mar. 2004.

[4] T.-M. Pan et al., "Study of high- $k \mathrm{Er}_{2} \mathrm{O}_{3}$ thin layers as ISFET sensitive insulator surface for $\mathrm{pH}$ detection," Sens. Actuators B, Chem., vol. 138, no. 2, pp. 619-624, May 2009

[5] H.-J. Jang and W.-J. Cho, "Fabrication of high-performance fully depleted silicon-on-insulator based dual-gate ion-sensitive field-effect transistor beyond the Nernstian limit," Appl. Phys. Lett., vol. 100, no. 7, p. 073701, Feb. 2010.

[6] H.-C. Lin et al., "Water passivation effect on polycrystalline silicon nanowires," Appl. Phys. Lett., vol. 91, no. 20, pp. 202113-1-202113-3, Nov. 2007.

[7] T.-M. Pan and K.-M. Liao, "Influence of oxygen content on the structural and sensing characteristics of $\mathrm{Y}_{2} \mathrm{O}_{3}$ sensing membrane for $\mathrm{pH}$-ISFET," Sens. Actuators B, Chem., vol. 128, no. 1, pp. 245-251, Dec. 2007.

[8] C. D. Fung, P. W. Cheung, and W. H. Ko, "A generalized theory of an electrolyte-insulator-semiconductor field-effect transistor," IEEE Trans. Electron Devices, vol. 33, no. 1, pp. 8-18, Jan. 1986.

[9] J.-L. Chiang et al., "Drift and hysteresis effects on $\mathrm{AlN} / \mathrm{SiO}_{2}$ gate pH ion-sensitive field-effect transistor," J. Appl. Phys., vol. 42, no. 8, pp. 4973-4977, Aug. 2003.

[10] O. Knopfmacher et al., "Nernst limit in dual-gated Si-nanowire FET sensors," Nano Lett., vol. 10, no. 6, pp. 2268-2274, May 2010.

[11] L. Bousse et al., "Comparison of the hysteresis of $\mathrm{Ta}_{2} \mathrm{O}_{5}$ and $\mathrm{Si}_{3} \mathrm{~N}_{4}$ pH-sensing insulators," Sens. Actuators B, Chem., vol. 17, no. 2, pp. 157-164, Jan. 1994.

[12] S. Jamasb, S. D. Collins, and R. L. Smith, "A physical model for threshold voltage instability in $\mathrm{Si}_{3} \mathrm{~N}_{4}$-gate $\mathrm{H}^{+}$-sensitive FET's (pH ISFET's)," IEEE Trans. Electron Devices, vol. 45, no. 6, pp. 1239-1245, Jun. 1998.

[13] S. Jamasb, "An analytical technique for counteracting drift in ionselective field effect transistors (ISFETs)," IEEE Sensors J., vol. 4, no. 6 , pp. 795-801, Dec. 2004.

[14] P. Hein and P. Egger, "Drift behaviour of ISFETs with $\mathrm{Si}_{3} \mathrm{~N}_{4} \mathrm{SiO}_{2}$ gate insulator," Sens. Actuators B, Chem., vol. 14, nos. 1-3, pp. 655-656, Jun. 1993.

[15] J.-L. Chiang et al., "Study on the temperature effect, hysteresis and drift of pH-ISFET devices based on amorphous tungsten oxide," Sens. Actuators B, Chem., vol. 76, nos. 1-3, pp. 624-628, Jun. 2001.

[16] J. C. Chou and Y. F. Wang, "Preparation and study on the drift and hysteresis properties of the tin oxide gate ISFET by the sol-gel method," Sens. Actuators B, Chem., vol. 86, no. 1, pp. 58-62, Aug. 2002. 